Repository of the Max Delbrück Center for Molecular Medicine (MDC) Berlin (Germany)

http://edoc.mdc-berlin.de/8868/

\title{
Cachexia has only one meaning
}

Friedrich C. Luft 


\title{
Cachexia has only one meaning
}

\author{
Friedrich C. Luft ${ }^{1}$ \\ ${ }^{1}$ HELIOS Kliniken-Berlin, Franz Volhard Clinic at the Max Delbrück Center Medical Faculty of the Charité, 13122 Berlin, \\ Germany
}

Cachexia means any general reduction in vitality and strength of body and mind resulting from any debilitating chronic disease. Cachexia is defined as loss of weight, muscle atrophy, fatigue, weakness, and loss of appetite in someone who is not actively trying to lose weight. These features markedly distinguish cachexia from starvation. Cachexia can be a sign of various underlying disorders. Physicians confronted with cachexia generally consider the possibility of cancer, certain infectious diseases such as tuberculosis or AIDS, parasitic diseases, autoimmune disorders, or chronic heart failure. Cachexia physically weakens patients to a state of immobility stemming from loss of appetite, asthenia, and anemia. The response to standard treatments is poor [1]. The above sounds straightforward enough. However, in PubMed, about 4,500 papers have been published on cachexia; interestingly, $>1,000$ of these papers are reviews. Such a relationship raises suspicion that little is known about the subject. Weber et al. [2], in this issue, introduce a novel inverse marker of clinical muscle wasting, namely, the measurement of plasma myoglobin concentration. Their report is immediately disconcerting, as the title of their paper ends in a question mark, implying a lack of selfconfidence on behalf of the authors. However, the entire cachexia field is riddled with question marks.

Weber et al. studied 17 cancer patients, the prototype patients exhibiting cachexia. The patients had lost $>20 \%$ of their body mass without intending to do so. A suitable matched control group of 27 subjects was recruited who had lost no weight. Plasma myoglobin, creatine kinase, quadriceps muscle cross-sectional area (by magnetic resonance imaging), muscle morphology from biopsies of the Vastus lateralis, body cell mass by impedance, and maximal oxygen uptake $\left(\mathrm{VO}_{2} \mathrm{max}\right)$ were all measured in these patients and control subjects. To no surprise, myoglobin, muscle cross-sectional area, body cell mass, and $\mathrm{VO}_{2}$ max were all lower in cachexic cancer patients than in healthy controls. However, in a multiple-regression analysis, myoglobin (hypomyoglobinemia) won out over other indicators $(27 \mu \mathrm{g} / \mathrm{dl}$ vs $42 \mu \mathrm{g} / \mathrm{dl})$; or did it? The authors found that myoglobin was directly correlated with cross-sectional muscle mass and was better than reduced creatine kinase in this regard, although the magnitude of the creatine kinase reduction was greater. The fact that creatine kinase and myoglobin were not elevated is of some mechanistic interest, since active muscle destruction would have featured elevation of both myoglobin and creatine kinase. Weber et al. [2] performed muscle biopsies on 11 patients and 15 control subjects. A marked size reduction in type 1 and 2 fibers occurred in the cachexic patients that corresponded to the reduction in cross-sectional area and correlated significantly with the reduced myoglobin levels.

What causes cachexia anyway? Are the diverse chronic (or not so chronic) cachexia conditions related? The cachexic patients presented by Weber et al. [2] all had cancer. Skipworth et al. [3] recently reviewed cancer cachexia. They stressed the role of host-tumor interaction, particularly pro-inflammatory cytokines. Tumor cells release cytokines and other factors locally to promote inflammation and thereby activate a local response. For instance, proteolysis-inducing factor (PIF) is a sulphated glycoprotein that produces muscle wasting in tumor-free mice when injected. The material has been identified in patients with pancreatic, breast, ovarian, lung, colon, rectum, and liver cancers. PIF may activate the ubiquitin-proteasome pathway by a nuclear factor kappaB (NF-KB) related mechanism. Lipid-mobilizing factor (LMF) is another cachexia protein produced by tumors. LMF increases lipid oxidation via induction of uncoupling protein expression, perhaps by interacting with the $\beta 3$ adrenoceptor.

Tumor products activate host cells. The activated host cells then initiate their own cytokine cascade, thereby inducing the hepatic acute phase protein response (APPR). APPR induction appears to be primarily an interleukin-6 (IL-6)-driven mechanism; however, tumor necrosis factor-alpha (TNF- $\alpha), \mathrm{IL}-2, \mathrm{IL}-8$, interferongamma (IFN- $\gamma$ ), parathyroid hormone-related peptide (PTHrP), and macrophage migratory inhibitory factor (MIF) also participate. Other cytokines such as IL-4, IL-10, and IL-13 have anti-inflammatory effects and are thought to repress cachexia. IL-15 has anabolic effects on skeletal muscle through the direct inhibition of muscle proteolysis.

The hepatic APPR includes a large number of "positive" acute-phase proteins, such as C-reactive protein (CRP) and fibrinogen. CRP correlates with weight loss, hypermetabolism, anorexia, and reduced survival. On the other hand, the circulating levels of "negative" acutephase proteins such as albumin decrease. Cancer cachexia is also associated with a neuroendocrine stress response. Weight-losing cancer patients exhibit insulin resistance, dysregulation of the autonomic nervous system, and sympathetic activation. A schematic suggested by Skipworth et al. [3] is given in Fig.1.

Another chronic cachexia condition is provoked by chronic heart failure (CHF). CHF patients who involuntarily lose non-edematous weight $>6 \%$ of their previous normal weight are at substantially greater risk for subsequent mortality [4]. The parameter has a very high sensitivityspecificity product. CHF cachexia correlates rather poorly with ejection fraction or the New York Heart Association functional cardiac classification. Proinflammatory cytokines, TNF- $\alpha, \mathrm{IL}-1, \mathrm{IL}-6$, and others again appear, as does the hepatic APPR. In CHF, there are no invading tumor cells that release mediators, although catecholamines, cortisol, natriuretic peptides, and heatshock proteins have been implicated. An imbalance between anabolic and catabolic pathways becomes established that involves numerous hormone systems, including neuropeptide $\mathrm{Y}$, insulin, cortisol, leptin, and ghrelin. A decrease in food intake alone does not trigger the wasting process. The initial triggers are unknown.

A related, more short-term condition developing over days to weeks is critical illness myopathy (CIM). This condition was first identified in intensive care unit patients who had 
developed the systemic inflammatory response syndrome (SIRS) usually in the framework of sepsis [5]. CIM patients commonly develop encephalopathy and neuropathy, as well as a diffuse, flaccid weakness of limbs and diaphragm. CIM is associated with SIRS, multiple organ failure, and corticosteroid and neuromuscular blocking agent administration. CIM features bioenergetic failure, mitochondrial dysfunction, inflammatory mediator-related proteolysis, hormonal dysregulation with glucose toxicity, myosin loss, and markedly increased total muscle catabolism. The triggers are unknown. The ubiquitin-proteosome pathway has been implicated along with cyclooxygenase activation, altered glucose transporter expression, MyoD suppression, impaired respiratory chain enzymes, ATP depletion, and insulin resistance. As mediators, TNF- $\alpha$, IFN- $y$, IL-1, IL-6, CRP, and the regulatory transcription factor NF-KB again prominently appear.

The cachexia syndromes are diverse diseases with certain features that suggest a final common pathway. The pathway leads to marked muscle wasting, systemic inflammation, hepatic APPR, and disturbed metabolism. The pathophysiological changes are tightly regulated by the same cytokines. Thus far, the final common pathway has not proved to be a therapeutic avenue. Elucidation of the triggers would appear more promising. The muscle biopsies performed by Weber et al. [2] could be helpful in this regard. Possibly, very early changes that could be identified by serial biopsy might be helpful. Such biopsies could be coupled with metabolic studies that can be discerned at the tissue level by microdialysis and similar techniques. Magnetic resonance spectroscopy coupled with imaging could be applied. The area is ripe for novel patient-oriented translational research.

Respectfully,

Friedrich C. Luft

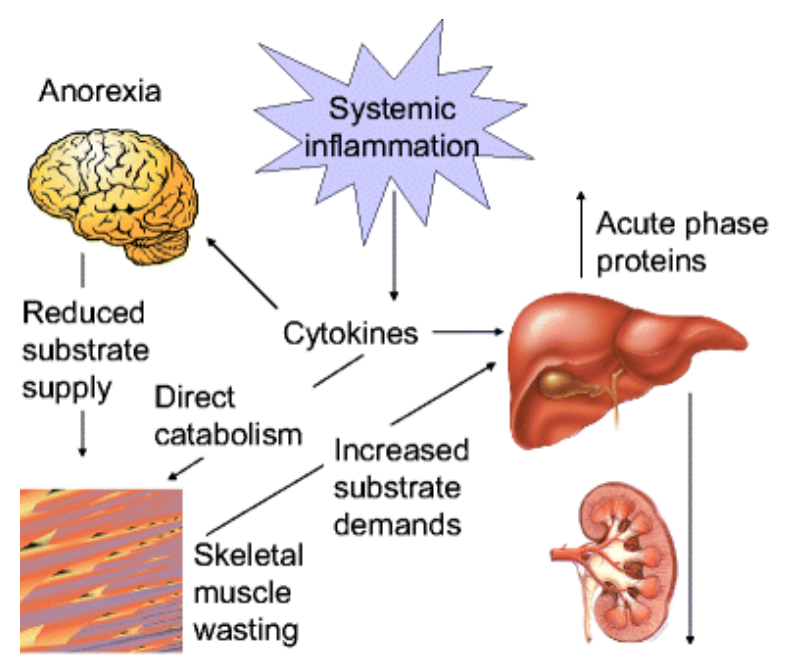

Urinary nitrogen loss

Fig.1: Pro-inflammatory cytokines induce muscle wasting either directly or indirectly. The process involves a cytokine storm, anorexia, metabolic disturbances, and enhanced catabolism, as well as acute-phase response proteins and their effects [3]

\section{Corresponding Author}

Friedrich C. Luft, luft@charite.de

\section{References}

1. Kotler DP (2000) Cachexia. Ann Intern Med 133:622-634.

2. Weber MA, Kinscherf R, Krakowski-Roosen $H$, Aulmann $M$, Renk H, Künkele A, Edler L, Kauczor HU, Hildebrant W (2007) Myoglobin plasma level related to muscle mass and fiber composition-a clinical marker of muscle wasting? J Mol Med.

3. Skipworth RJE, Stewart GD, Dejong CHC, Preston T, Fearon $\mathrm{KCH}$ (2007) Pathophysiology of cancer cachexia: much more than a host-tumour interaction? Clin Nutr.

4. Von Haehling S, Doehner W, Anker SD (2007) Nutrition, metabolism, and the complex pathophysiology of cachexia in chronic heart failure. Cardiovasc Res 73:298-309.

5. Friedrich $O$ (2006) Critical illness myopathy: what is happening? Curr Opin Clin Nutr Metab Care 9:403-409. 